\title{
Partial automorphisms and injective partial endomorphisms of a finite undirected path
}

\author{
I. Dimitrova ${ }^{2}$ - V. H. Fernandes ${ }^{3}$ J. Koppitz ${ }^{1}$ - T. M. Quinteiro ${ }^{4,5}$
}

Received: 27 January 2021 / Accepted: 20 April 2021 / Published online: 18 May 2021

(c) The Author(s), under exclusive licence to Springer Science+Business Media, LLC, part of Springer Nature 2021

\begin{abstract}
In this paper, we study partial automorphisms and, more generally, injective partial endomorphisms of a finite undirected path from Semigroup Theory perspective. Our main objective is to give formulas for the ranks of the monoids $\operatorname{IEnd}\left(P_{n}\right)$ and $\operatorname{PAut}\left(P_{n}\right)$ of all injective partial endomorphisms and of all partial automorphisms of the undirected path $P_{n}$ with $n$ vertices. We also describe Green's relations of PAut $\left(P_{n}\right)$ and $\operatorname{IEnd}\left(P_{n}\right)$ and calculate their cardinals.
\end{abstract}

Keywords Injective partial endomorphisms · Partial automorphisms · Paths · Generators · Rank

Mathematics Subject Classification 05C38 $20 \mathrm{M} 10 \cdot 20 \mathrm{M} 20 \cdot 05 \mathrm{C} 25$

\section{Introduction and preliminaries}

As well as automorphisms of graphs allow one to establish natural connections between Graph Theory and Group Theory, endomorphisms of graphs allow similar connections between Graph Theory and Semigroup Theory. Likewise, in particular, partial automorphisms of graphs relate Graph Theory with Inverse Semigroup Theory. This has led, over the last decades, many authors to become interested in the study of combinatorial and algebraic properties of monoids of endomorphisms of graphs. One of the most studied algebraic notions is regularity, in the sense of Semigroup Theory. A general solution to the problem, posed in 1987 by Knauer and Wilkeit, see [29], of

Communicated by László Márki.

This work is funded by national funds through the FCT - Fundação para a Ciência e a Tecnologia, I.P., under the scope of the project UIDB/00297/2020 (Center for Mathematics and Applications).

$凶 \quad$ J. Koppitz

koppitz@math.bas.bg

Extended author information available on the last page of the article 
which graphs have a regular monoid of endomorphisms has proved to be very difficult to obtain. Despite that, various authors studied and solved this question for some special classes of graphs (see, for instance, [7-9,16-18,20,21,25-28,31,32]).

The rank of a monoid $S$, denoted by rank $S$, is the least number of generators of $S$. In this paper, we focus our attention on this important notion of Semigroup Theory, which has been, in recent years, the subject of intensive research.

Let $\Omega$ be a finite set with at least 3 elements. It is well known that the symmetric group $\mathscr{S}(\Omega)$ of $\Omega$ has rank 2 (as a semigroup, a monoid or a group). Furthermore, the monoid of all transformations $\mathscr{T}(\Omega)$ of $\Omega$, the monoid of all partial transformations $\mathscr{P} \mathscr{T}(\Omega)$ of $\Omega$ and the symmetric inverse monoid $\mathscr{I}(\Omega)$ of $\Omega$ have ranks 3, 4, and 3, respectively. The survey [10] presents these results and similar ones for other classes of transformation monoids, in particular, for monoids of order-preserving transformations and for some of their extensions. More recently, for instance, the papers $[1,2,5,11-15,23,33,34]$ are dedicated to the computation of the ranks of certain (classes of transformation) semigroups or monoids.

Now, let $G=(V, E)$ be a simple graph (i.e. an undirected graph without loops and without multiple edges). Let $\alpha$ be a partial transformation of $V$. Denote by Dom $\alpha$ the domain of $\alpha$ and by $\operatorname{Im} \alpha$ the image of $\alpha$. We say that $\alpha$ is:

- A partial endomorphism of $G$ if $\{u, v\} \in E$ implies $\{u \alpha, v \alpha\} \in E$, for all $u, v \in$ $\operatorname{Dom} \alpha$;

- A partial automorphism of $G$ if $\alpha$ is an injective mapping (i.e. a partial permutation) and $\alpha$ and $\alpha^{-1}$ are both partial endomorphisms.

If $\alpha$ is a full mapping (i.e. $\alpha \in \mathscr{T}(V)$ ) then a partial endomorphism (respectively, partial automorphism) is just called endomorphism (respectively, automorphism).

Notice that, for finite graphs, any bijective endomorphism is an automorphism.

Denote by:

- PEnd $(G)$ the set of all partial endomorphisms of $G$;

- $\operatorname{End}(G)$ the set of all endomorphisms of $G$;

- IEnd $(G)$ the set of all injective partial endomorphisms of $G$;

- PAut $(G)$ the set of all partial automorphisms of $G$;

- Aut $(G)$ the set of all automorphisms of $G$.

Clearly, $\operatorname{PEnd}(G), \operatorname{End}(G), \operatorname{IEnd}(G), \operatorname{PAut}(G)$, and $\operatorname{Aut}(G)$ are monoids under composition of maps with the identity mapping id as the identity element. Moreover, $\operatorname{Aut}(G)$ is also a group and $\operatorname{PAut}(G)$ is an inverse semigroup: $\operatorname{Aut}(G) \subseteq \mathscr{S}(V)$ and $\operatorname{PAut}(G) \subseteq \mathscr{I}(V)$. It is also clear that

$$
\operatorname{Aut}(G) \subseteq \operatorname{End}(G) \subseteq \operatorname{PEnd}(G)
$$

and

$$
\operatorname{Aut}(G) \subseteq \operatorname{PAut}(G) \subseteq \operatorname{IEnd}(G) \subseteq \operatorname{PEnd}(G)
$$

(these inclusions may not be strict). 
Let $\mathbb{N}$ be the set of all positive integers and let $n \in \mathbb{N}$. Let $P_{n}$ be the undirected path with $n$ vertices. Notice that we may take

$$
P_{n}=(\{1, \ldots, n\},\{\{i, i+1\} \mid i=1, \ldots, n-1\}) .
$$

The number of endomorphisms of $P_{n}$ has been determined by Arworn [3] (see also the paper [30] by Michels and Knauer). In addition, several other combinatorial and algebraic properties of $P_{n}$ were also studied in these two papers and also, for instance, in $[4,19]$. The authors in [6] studied several properties of the monoid $\operatorname{End}\left(P_{n}\right)$. In particular, they characterized regular elements and determined the rank of $\operatorname{End}\left(P_{n}\right)$.

The main objective of the present paper is to determine the ranks of the monoids $\operatorname{PAut}\left(P_{n}\right)$ and $\operatorname{IEnd}\left(P_{n}\right)$. We will show that

$$
\operatorname{rank} \operatorname{PAut}\left(P_{n}\right)=\left\{\begin{array}{ll}
2 & \text { for } n=1 \\
2 & \text { for } n=2 \\
3 & \text { for } n=3 \\
n-1 & \text { for } n \geq 4
\end{array} \text { and } \quad \operatorname{rank} \operatorname{IEnd}\left(P_{n}\right)= \begin{cases}2 & \text { for } n=1 \\
2 & \text { for } n=2 \\
4 & \text { for } n=3 \\
n+\left\lceil\frac{n}{2}\right\rceil-2 & \text { for } n \geq 4\end{cases}\right.
$$

We also aim to describe Green's relations of PAut $\left(P_{n}\right)$ and IEnd $\left(P_{n}\right)$ and to calculate the cardinals of both monoids.

Observe that PAut $\left(P_{n}\right)$ and $\operatorname{IEnd}\left(P_{n}\right)$ are submonoids of the symmetric inverse monoid $\mathscr{I}_{n}=\mathscr{I}(\{1, \ldots, n\})$.

Recall that the Green's relations $\mathscr{L}, \mathscr{R}$, and $\mathscr{J}$ of a monoid $S$ are defined as following: for $\alpha, \beta \in S$,

$-\alpha \mathscr{L} \beta$ if and only if there exist $\gamma, \delta \in S$ such that $\alpha=\gamma \beta$ and $\beta=\delta \alpha$;

$-\alpha \mathscr{R} \beta$ if and only if there exist $\gamma^{\prime}, \delta^{\prime} \in S$ such that $\alpha=\beta \gamma^{\prime}$ and $\beta=\alpha \delta^{\prime}$;

$-\alpha \mathscr{J} \beta$ if and only if there exist $\gamma, \gamma^{\prime}, \delta, \delta^{\prime} \in S$ such that $\alpha=\gamma \beta \gamma^{\prime}$ and $\beta=\delta \alpha \delta^{\prime}$.

The relations $\mathscr{L}$ and $\mathscr{R}$ commute (i.e. $\mathscr{L} \circ \mathscr{R}=\mathscr{R} \circ \mathscr{L}$ ) and Green's relation $\mathscr{D}$ is defined by $\mathscr{D}=\mathscr{L} \circ \mathscr{R}=\mathscr{R} \circ \mathscr{L}$ (i.e. $\alpha \mathscr{D} \beta$ if and only if there exists $\sigma \in S$ such that $\alpha \mathscr{L} \sigma \mathscr{R} \beta$, for $\alpha, \beta \in S$ ). Notice that for a finite monoid the relations $\mathscr{J}$ and $\mathscr{D}$ coincide. Finally, we have Green's relation $\mathscr{H}$ defined by $\mathscr{H}=\mathscr{L} \cap \mathscr{R}$.

If $S$ is an inverse semigroup of injective partial transformations on a given set, then the relations $\mathscr{L}, \mathscr{R}$, and $\mathscr{H}$ can be described as following: for $\alpha, \beta \in S$,

$-\alpha \mathscr{L} \beta$ if and only if $\operatorname{Im} \alpha=\operatorname{Im} \beta$;

$-\alpha \mathscr{R} \beta$ if and only if $\operatorname{Dom} \alpha=\operatorname{Dom} \beta$;

$-\alpha \mathscr{H} \beta$ if and only if $\operatorname{Im} \alpha=\operatorname{Im} \beta$ and $\operatorname{Dom} \alpha=\operatorname{Dom} \beta$.

Since PAut $\left(P_{n}\right)$ is an inverse semigroup, it remains to obtain a description of its Green's relation $\mathscr{J}$. On the other hand, that is not the situation of $\operatorname{IEnd}\left(P_{n}\right)$, for $n \geq 3$, since IEnd $\left(P_{n}\right)$ is not an inverse semigroup (for instance, $\left(\begin{array}{ll}1 & 3 \\ 1 & 2\end{array}\right) \in \operatorname{IEnd}\left(P_{n}\right)$ is not a regular element of $\left.\operatorname{IEnd}\left(P_{n}\right)\right)$. Notice that $\operatorname{IEnd}\left(P_{n}\right)=\operatorname{PAut}\left(P_{n}\right)$, for $n=1,2$.

For general background on Semigroup Theory and standard notation, we refer the reader to Howie's book [22]. Regarding Algebraic Graph Theory, our main reference is Knauer's book [24]. 


\section{Green's relations}

Let $n \in \mathbb{N}$. We now describe Green's relations $\mathscr{L}, \mathscr{R}, \mathscr{H}$, and $\mathscr{J}$ of the monoid $\operatorname{IEnd}\left(P_{n}\right)$ as well as Green's relation $\mathscr{J}$ of the inverse monoid $\operatorname{PAut}\left(P_{n}\right)$.

In this section, for a set $X \subseteq \mathbb{N}$, we need the following concept. A set $I \subseteq X$ is called a maximal interval of $X$ if $I$ satisfies the following properties:

- $I$ is an interval of $X$ (i.e. $x, y \in I$ and $z \in \mathbb{N}$ with $x<z<y$ implies $z \in I$ );

- If $J \subseteq X$ is an interval of $X$ then $I \subseteq J$ implies $I=J$.

Recall that a partial transformation $\alpha$ of $\{1, \ldots, n\}$ is said to be order-preserving (respectively, order-reversing) if $x<y$ implies $x \alpha \leq y \alpha$ (respectively, if $x<y$ implies $x \alpha \geq y \alpha$ ), for all $x, y \in \operatorname{Dom} \alpha$.

Let $\alpha \in \mathscr{I}_{n}$. The following observations are easy to show:

$-\alpha \in \operatorname{IEnd}\left(P_{n}\right)$ if and only if for each interval $I$ of Dom $\alpha$ the image $I \alpha$ is an interval of $\operatorname{Im} \alpha$;

- If $\alpha \in \operatorname{IEnd}\left(P_{n}\right)$ then $\alpha$ is order-preserving or order-reversing in $I$ (i.e. the restriction $\left.\alpha\right|_{I}$ of $\alpha$ to $I$ is an order-preserving or order-reversing transformation), for each interval $I$ of $\operatorname{Dom} \alpha$;

- If for each maximal interval $I$ of Dom $\alpha$ the image $I \alpha$ is an interval of $\operatorname{Im} \alpha$ and $\alpha$ is order-preserving or order-reversing in $I$ then $\alpha \in \operatorname{IEnd}\left(P_{n}\right)$;

- If $\alpha \in \operatorname{PAut}\left(P_{n}\right)$ and $I$ is a maximal interval of Dom $\alpha$ then the image $I \alpha$ is a maximal interval of $\operatorname{Im} \alpha$;

- If for each maximal interval $I$ of Dom $\alpha$ the image $I \alpha$ is a maximal interval of $\operatorname{Im} \alpha$ and $\alpha$ is order-preserving or order-reversing in $I$ then $\alpha \in \operatorname{PAut}\left(P_{n}\right)$.

Let $\alpha \in \mathscr{I}_{n}$. Let $\left\{X_{1}, \ldots, X_{k}\right\}$ be a partition of Dom $\alpha$. We will use the notation $\alpha=\left(\begin{array}{ccc}X_{1} & \cdots & X_{k} \\ Y_{1} & \cdots & Y_{k}\end{array}\right)$ to express that $Y_{i}=\left(X_{i}\right) \alpha$, for $i \in\{1, \ldots, k\}$.

Let $\alpha, \beta \in \operatorname{IEnd}\left(P_{n}\right)$. Since IEnd $\left(P_{n}\right)$ is a submonoid of the inverse monoid $\mathscr{I}_{n}$, if $\alpha \mathscr{L} \beta$ (respectively, $\alpha \mathscr{R} \beta$ ) in IEnd $\left(P_{n}\right)$ then $\alpha \mathscr{L} \beta$ (respectively, $\alpha \mathscr{R} \beta$ ) in $\mathscr{I}_{n}$, whence $\operatorname{Im} \alpha=\operatorname{Im} \beta$ (respectively, $\operatorname{Dom} \alpha=\operatorname{Dom} \beta$ ). Moreover, we have the following descriptions of the relations $\mathscr{L}$ and $\mathscr{R}$ in $\operatorname{IEnd}\left(P_{n}\right)$ :

Proposition 1 Let $\alpha, \beta \in \operatorname{IEnd}\left(P_{n}\right)$ and let $\left\{I_{1}, I_{2}, \ldots, I_{k}\right\}$ and $\left\{I_{1}^{\prime}, I_{2}^{\prime}, \ldots, I_{l}^{\prime}\right\}$ be the (partitions into) maximal intervals of $\operatorname{Dom} \alpha$ and in $\operatorname{Dom} \beta$, respectively. Then, the following three conditions are equivalent:

1. $\alpha \mathscr{L} \beta$;

2. $\left\{I_{1} \alpha, I_{2} \alpha, \ldots, I_{k} \alpha\right\}=\left\{I_{1}^{\prime} \beta, I_{2}^{\prime} \beta, \ldots, I_{l}^{\prime} \beta\right\}$;

3. $\operatorname{Im} \alpha=\operatorname{Im} \beta$ and $\alpha \beta^{-1} \in \operatorname{PAut}\left(P_{n}\right)$.

Proof [1 $\Rightarrow 2$ ] Suppose that $\alpha \mathscr{L} \beta$. Then, by the definition of Green's relation $\mathscr{L}$, there exist $\gamma, \delta \in \operatorname{IEnd}\left(P_{n}\right)$ such that $\alpha=\gamma \beta$ and $\beta=\delta \alpha$. Let $i \in\{1, \ldots, k\}$. Since Dom $\alpha \subseteq \operatorname{Dom} \gamma, I_{i}$ is also an interval of Dom $\gamma$, whence $I_{i} \gamma$ is an interval of Dom $\beta$ and so $I_{i} \gamma \subseteq I_{j}^{\prime}$, for some $j \in\{1, \ldots, l\}$. It follows that $I_{i} \alpha=I_{i} \gamma \beta \subseteq I_{j}^{\prime} \beta$, for some $j \in\{1, \ldots, l\}$. Similarly, we may show that, for all $j \in\{1, \ldots, l\}$, there exists $i \in\{1, \ldots, k\}$ such that $I_{j}^{\prime} \beta \subseteq I_{i} \alpha$. Now, since $\operatorname{Im} \alpha=\operatorname{Im} \beta$, we may deduce that $\left\{I_{1} \alpha, I_{2} \alpha, \ldots, I_{k} \alpha\right\}=\left\{I_{1}^{\prime} \beta, I_{2}^{\prime} \beta, \ldots, I_{l}^{\prime} \beta\right\}$. 
$[2 \Rightarrow 3]$ From $\left\{I_{1} \alpha, I_{2} \alpha, \ldots, I_{k} \alpha\right\}=\left\{I_{1}^{\prime} \beta, I_{2}^{\prime} \beta, \ldots, I_{l}^{\prime} \beta\right\}$ it follows immediately that $k=l$ and $\operatorname{Im} \alpha=\operatorname{Im} \beta$. Let $\sigma$ be the permutation of $\{1, \ldots, k\}$ such that $I_{i} \alpha=$ $I_{i \sigma}^{\prime} \beta$, for all $i \in\{1, \ldots, k\}$. Then $\alpha \beta^{-1}=\left(\begin{array}{cccc}I_{1} & I_{2} & \cdots & I_{k} \\ I_{1 \sigma}^{\prime} & I_{2 \sigma}^{\prime} & \cdots & I_{k \sigma}^{\prime}\end{array}\right)$ and so $\alpha \beta^{-1}$ maps maximal intervals of its domain into maximal intervals of its image. Hence, in order to prove that $\alpha \beta^{-1} \in \operatorname{PAut}\left(P_{n}\right)$, it suffices to show that $\alpha \beta^{-1}$ is order-preserving or order-reversing in $I_{i}$, for $i \in\{1, \ldots, k\}$. Let $i \in\{1, \ldots, k\}$. Then, we have $\left.\alpha \beta^{-1}\right|_{I_{i}}=$ $\left.\left.\alpha\right|_{I_{i}} \beta^{-1}\right|_{I_{i} \alpha}=\left.\left.\alpha\right|_{I_{i}} \beta^{-1}\right|_{I_{i \sigma}^{\prime} \beta}$ and $\left.\beta^{-1}\right|_{I_{i \sigma}^{\prime} \beta}=\left(\begin{array}{c}I_{i \sigma}^{\prime} \beta \\ I_{i \sigma}^{\prime}\end{array}\right)$. As $I_{i}$ is an interval, $\left.\alpha\right|_{I_{i}}$ is order-preserving or order-reversing. On the other hand, as $I_{i \sigma}^{\prime}$ is an interval, $\left.\beta\right|_{I_{i \sigma}^{\prime}}$ is order-preserving or order-reversing and so its inverse mapping $\left.\beta^{-1}\right|_{I_{i \sigma}^{\prime} \beta}$ is also orderpreserving or order-reversing. Thus, $\alpha \beta^{-1}$ is order-preserving or order-reversing in $I_{i}$, as required.

[3 $\Rightarrow 1]$ From $\operatorname{Im} \alpha=\operatorname{Im} \beta$ and $\alpha \beta^{-1} \in \operatorname{PAut}\left(P_{n}\right)$, it follows that $\alpha \beta^{-1}$ and $\beta \alpha^{-1}=\left(\alpha \beta^{-1}\right)^{-1}$ lie in $\operatorname{IEnd}\left(P_{n}\right),\left(\alpha \beta^{-1}\right) \beta=\alpha\left(\beta^{-1} \beta\right)=\alpha$ id $\left.\right|_{\operatorname{Im} \beta}=\alpha$ id $\left.\right|_{\operatorname{Im} \alpha}=$ $\alpha$ and $\left(\alpha \beta^{-1}\right)^{-1} \alpha=\left(\beta \alpha^{-1}\right) \alpha=\beta\left(\alpha^{-1} \alpha\right)=\beta$ id $\left.\right|_{\operatorname{Im} \alpha}=\beta$ id $\left.\right|_{\operatorname{Im} \beta}=\beta$, whence $\alpha \mathscr{L} \beta$.

Proposition 2 Let $\alpha, \beta \in \operatorname{IEnd}\left(P_{n}\right)$. Then $\alpha \mathscr{R} \beta$ if and only if $\operatorname{Dom} \alpha=\operatorname{Dom} \beta$ and $\alpha^{-1} \beta \in \operatorname{PAut}\left(P_{n}\right)$.

Proof Suppose that $\alpha \mathscr{R} \beta$. Then Dom $\alpha=\operatorname{Dom} \beta$. Moreover, there exist transformations $\gamma, \delta \in \operatorname{IEnd}\left(P_{n}\right)$ such that $\beta=\alpha \gamma$ and $\alpha=\beta \delta$. Then, we have $\alpha^{-1} \beta=$ $\alpha^{-1} \alpha \gamma=\left.\mathrm{id}\right|_{\operatorname{Im} \alpha} \gamma=\left.\gamma\right|_{\operatorname{Im} \alpha}$ and $\left(\alpha^{-1} \beta\right)^{-1}=\beta^{-1} \alpha=\beta^{-1} \beta \delta=\left.\mathrm{id}\right|_{\operatorname{Im} \beta} \delta=\left.\delta\right|_{\operatorname{Im} \beta}$. Since, clearly, any restriction of a transformation of $\operatorname{IEnd}\left(P_{n}\right)$ is still a transformation of IEnd $\left(P_{n}\right)$, we have $\alpha^{-1} \beta, \beta^{-1} \alpha \in \operatorname{IEnd}\left(P_{n}\right)$ and so $\alpha^{-1} \beta \in \operatorname{PAut}\left(P_{n}\right)$.

Conversely, admit that $\operatorname{Dom} \alpha=\operatorname{Dom} \beta$ and $\alpha^{-1} \beta \in \operatorname{PAut}\left(P_{n}\right)$. Then $\alpha^{-1} \beta$, $\beta^{-1} \alpha=\left(\alpha^{-1} \beta\right)^{-1} \in \operatorname{IEnd}\left(P_{n}\right), \beta=\left.\mathrm{id}\right|_{\operatorname{Dom} \beta} \beta=\left.\mathrm{id}\right|_{\operatorname{Dom} \alpha} \beta=\left(\alpha \alpha^{-1}\right) \beta=\alpha\left(\alpha^{-1} \beta\right)$ and $\alpha=\left.\mathrm{id}\right|_{\operatorname{Dom} \alpha} \alpha=\left.\mathrm{id}\right|_{\operatorname{Dom} \beta} \alpha=\left(\beta \beta^{-1}\right) \alpha=\beta\left(\beta^{-1} \alpha\right)$, whence $\alpha \mathscr{R} \beta$, as required.

Since $\mathscr{H}=\mathscr{R} \cap \mathscr{L}$, it follows immediately that:

Corollary 1 Let $\alpha, \beta \in \operatorname{IEnd}\left(P_{n}\right)$. Then $\alpha \mathscr{H} \beta$ if and only if $\operatorname{Dom} \alpha=\operatorname{Dom} \beta, \operatorname{Im} \alpha=$ $\operatorname{Im} \beta$ and $\alpha^{-1} \beta, \alpha \beta^{-1} \in \operatorname{PAut}\left(P_{n}\right)$.

Before presenting our descriptions of Green's relation $\mathscr{J}$ on $\operatorname{IEnd}\left(P_{n}\right)$ and on PAut $\left(P_{n}\right)$, we need to introduce some notions and notations.

For $A, B \subseteq \mathbb{N}$, denote by $A<B$ if $a<b$ for all $a \in A$ and $b \in B$.

Let $a=\left(a_{1}, \ldots, a_{p}\right)$ be a sequence of elements of $\mathbb{N}$. We define the reverse of $a$ as being the sequence $a^{R}=\left(a_{p}, \ldots, a_{1}\right)$.

Let $\alpha \in \operatorname{IEnd}\left(P_{n}\right)$ and let $J$ be a maximal interval of $\operatorname{Im} \alpha$. Define the type of $J$ to be the sequence $\tau_{\alpha}(J)=\left(\left|I_{1}\right|,\left|I_{2}\right|, \ldots,\left|I_{p}\right|\right)$, where $\left\{I_{1}, I_{2}, \ldots, I_{p}\right\}$ are the maximal intervals of $J \alpha^{-1}$ such that $I_{i} \alpha<I_{i+1} \alpha$, for $1 \leq i<p$.

Now, let $\alpha, \beta \in \operatorname{IEnd}\left(P_{n}\right)$. We say that $\alpha$ and $\beta$ have similar type if there exists a bijection $\sigma$ from the set of maximal intervals of $\operatorname{Im} \alpha$ into the set of maximal intervals of $\operatorname{Im} \beta$ such that $\tau_{\alpha}(J) \in\left\{\tau_{\beta}(J \sigma), \tau_{\beta}(J \sigma)^{R}\right\}$, for any maximal interval $J$ of $\operatorname{Im} \alpha$. 
Observe that two elements $\alpha$ and $\beta$ of $\operatorname{IEnd}\left(P_{n}\right)$ have similar type if and only if they have maximal intervals of their images with the same type up to reversion and the same number of occurrences.

Lemma 1 Let $\alpha, \beta \in \operatorname{IEnd}\left(P_{n}\right)$ be such that $\alpha$ and $\beta$ have similar type. Then, there exist $\gamma, \delta \in \operatorname{PAut}\left(P_{n}\right)$ such that $\beta=\gamma \alpha \delta$ and $\alpha=\gamma^{-1} \beta \delta^{-1}$.

Proof Let $\left\{J_{1}, J_{2}, \ldots, J_{k}\right\}$ and $\left\{J_{1}^{\prime}, J_{2}^{\prime}, \ldots, J_{k}^{\prime}\right\}$ be the maximal intervals of $\operatorname{Im} \alpha$ and $\operatorname{Im} \beta$, respectively. Then there exist a permutation $\sigma$ of $\{1, \ldots, k\}$ such that $\tau_{\beta}\left(J_{r}^{\prime}\right) \in$ $\left\{\tau_{\alpha}\left(J_{r \sigma}\right), \tau_{\alpha}\left(J_{r \sigma}\right)^{R}\right\}$, for $r=1, \ldots, k$.

For $1 \leq r \leq k$, let $\left\{I_{r, 1}^{\prime}, I_{r, 2}^{\prime}, \ldots, I_{r, p_{r}}^{\prime}\right\}$ and $\left\{I_{r \sigma, 1}, I_{r \sigma, 2}, \ldots, I_{r \sigma, p_{r}}\right\}$ be the maximal intervals of $J_{r}^{\prime} \beta^{-1}$ and $J_{r \sigma} \alpha^{-1}$, respectively, such that $I_{r, i}^{\prime} \beta<I_{r, i+1}^{\prime} \beta$ and $I_{r \sigma, i} \alpha<I_{r \sigma, i+1} \alpha$, for all $1 \leq i<p_{r}$. Moreover, let $J_{r, i}^{\prime}=I_{r, i}^{\prime} \beta$ and $J_{r \sigma, i}=I_{r \sigma, i} \alpha$, for $r=1, \ldots, k$ and $i=1, \ldots, p_{r}$. Clearly, $J_{r}^{\prime}=J_{r, 1}^{\prime} \cup J_{r, 2}^{\prime} \cup \cdots \cup J_{r, p_{r}}^{\prime}$ and $J_{r \sigma}=J_{r \sigma, 1} \cup J_{r \sigma, 2} \cup \cdots \cup J_{r \sigma, p_{r}}$.

Let $r=1, \ldots, k$. We define partial transformations $\gamma_{r}$ and $\delta_{r}$ as following:

- Dom $\gamma_{r}=\cup\left\{I_{r, 1}^{\prime}, I_{r, 2}^{\prime}, \ldots, I_{r, p_{r}}^{\prime}\right\}=J_{r}^{\prime} \beta^{-1}$;

- Dom $\delta_{r}=\cup\left\{J_{r \sigma, 1}, J_{r \sigma, 2}, \ldots, J_{r \sigma, p_{r}}\right\}=J_{r \sigma}$;

$-I_{r, i}^{\prime} \gamma_{r}= \begin{cases}I_{r \sigma, i} & \text { if } \tau_{\beta}\left(J_{r}^{\prime}\right)=\tau_{\alpha}\left(J_{r \sigma}\right) \\ I_{r \sigma, p_{r}-i+1} & \text { if } \tau_{\beta}\left(J_{r}^{\prime}\right)=\tau_{\alpha}\left(J_{r \sigma}\right)^{R},\end{cases}$

for $i=1, \ldots, p_{r}$;

$-J_{r \sigma, i} \delta_{r}= \begin{cases}J_{r, i}^{\prime} & \text { if } \tau_{\beta}\left(J_{r}^{\prime}\right)=\tau_{\alpha}\left(J_{r \sigma}\right) \\ J_{r, p_{r}-i+1}^{\prime} & \text { if } \tau_{\beta}\left(J_{r}^{\prime}\right)=\tau_{\alpha}\left(J_{r \sigma}\right)^{R},\end{cases}$

for $i=1, \ldots, p_{r}$;

$-\left.\gamma_{r}\right|_{I_{r, i}^{\prime}}$ is $\left\{\begin{array}{l}\text { order-preserving if (a) or (b) is satisfied } \\ \text { order-reversing otherwise, }\end{array}\right.$

where

(a) $\tau_{\beta}\left(J_{r}^{\prime}\right)=\tau_{\alpha}\left(J_{r \sigma}\right)$ and $\left.\alpha\right|_{I_{r \sigma, i}}$ and $\left.\beta\right|_{I_{r, i}^{\prime}}$ are both order-preserving or both order-reversing, and

(b) $\tau_{\beta}\left(J_{r}^{\prime}\right)=\tau_{\alpha}\left(J_{r \sigma}\right)^{R}$ and $\left.\alpha\right|_{I_{r \sigma, p_{r}-i+1}}$ is order-preserving and $\left.\beta\right|_{I_{r, i}^{\prime}}$ is orderreversing or vice versa,

for $i=1, \ldots, p_{r}$;

$-\left.\delta_{r}\right|_{J_{r \sigma}}$ is $\left\{\begin{array}{l}\text { order-preserving if } \tau_{\beta}\left(J_{r}^{\prime}\right)=\tau_{\alpha}\left(J_{r \sigma}\right) \\ \text { order-reversing if } \tau_{\beta}\left(J_{r}^{\prime}\right)=\tau_{\alpha}\left(J_{r \sigma}\right)^{R} .\end{array}\right.$

It is easy to verify that both $\gamma_{r}$ and $\delta_{r}$ are well defined. Then, we define partial transformations $\gamma$ and $\delta$ as follows:

- Dom $\gamma=\cup\left\{I_{1,1}^{\prime}, \ldots, I_{1, p_{1}}^{\prime}, \ldots, I_{k, 1}^{\prime}, \ldots, I_{k, p_{k}}^{\prime}\right\}=\operatorname{Dom} \beta$;

- Dom $\delta=\cup\left\{J_{1 \sigma, 1}, \ldots, J_{1 \sigma, p_{1}}, \ldots, J_{k \sigma, 1}, \ldots, J_{k \sigma, p_{k}}\right\}=\operatorname{Im} \alpha$;

$-\left.\gamma\right|_{I_{r, s}^{\prime}}=\left.\gamma_{r}\right|_{I_{r, s}^{\prime}}$ for $r=1, \ldots, k$ and $s=1, \ldots, p_{r}$;

$-\left.\delta\right|_{J_{r \sigma, s}}=\left.\delta_{r}\right|_{J_{r \sigma, s}}$ for $r=1, \ldots, k$ and $s=1, \ldots, p_{r}$.

Clearly, both transformations $\gamma$ and $\delta$ are partial automorphisms. Let $r=1, \ldots, k$ and $s=1, \ldots, p_{r}$. Then

$$
I_{r, s}^{\prime} \gamma \alpha \delta=\left\{\begin{array}{l}
I_{r \sigma, s} \alpha \delta=\quad J_{r \sigma, s} \delta=J_{r, s}^{\prime}=I_{r, s}^{\prime} \beta \text { if } \tau_{\beta}\left(J_{r}^{\prime}\right)=\tau_{\alpha}\left(J_{r \sigma}\right) \\
I_{r \sigma, p_{r}-s+1} \alpha \delta=J_{r \sigma, p_{r}-s+1} \delta=J_{r, s}^{\prime}=I_{r, s}^{\prime} \beta \text { if } \tau_{\beta}\left(J_{r}^{\prime}\right)=\tau_{\alpha}\left(J_{r \sigma}\right)^{R} .
\end{array}\right.
$$


Taking into account properties (a) and (b), we can deduce that $\left.\gamma \alpha \delta\right|_{I_{r, s}^{\prime}}$ is orderpreserving if $\left.\beta\right|_{I_{r, s}^{\prime}}$ is order-preserving and $\left.\gamma \alpha \delta\right|_{I_{r, s}^{\prime}}$ is order-reversing if $\left.\beta\right|_{I_{r, s}^{\prime}} ^{\prime}$ is orderreversing, which allows us to conclude that $\beta=\gamma \alpha \delta$. On the other hand, since $\operatorname{Im} \gamma=\operatorname{Dom} \alpha$ and $\operatorname{Im} \alpha=\operatorname{Dom} \delta$, we obtain $\gamma^{-1} \gamma \alpha \delta \delta^{-1}=\left.\mathrm{id}\right|_{\operatorname{Dom} \alpha} \alpha$ id $\left.\right|_{\operatorname{Im} \alpha}=\alpha$ and so we also have $\alpha=\gamma^{-1} \beta \delta^{-1}$, as required.

Now, we can describe Green's relation $\mathscr{J}$ for the monoid IEnd $\left(P_{n}\right)$.

Proposition 3 Let $\alpha, \beta \in \operatorname{IEnd}\left(P_{n}\right)$. Then $\alpha \mathscr{J} \beta$ if and only if $\alpha$ and $\beta$ have similar type.

Proof Let $\alpha, \beta \in \operatorname{IEnd}\left(P_{n}\right)$ be such that $\alpha \mathscr{J} \beta$. Then, there exists $\gamma \in \operatorname{IEnd}\left(P_{n}\right)$ such that $\alpha \mathscr{L} \gamma \mathscr{R} \beta$ and so, by Propositions 1 and 2 , we have $\operatorname{Dom} \gamma=\operatorname{Dom} \beta$ and $\operatorname{Im} \gamma=$ $\operatorname{Im} \alpha$ and $\alpha \gamma^{-1}, \gamma^{-1} \beta \in \operatorname{PAut}\left(P_{n}\right)$. In addition, $\alpha^{-1} \alpha \gamma^{-1}=\gamma^{-1}=\gamma^{-1} \beta \beta^{-1}$. Moreover, $\operatorname{Dom}\left(\gamma^{-1} \beta\right)=\operatorname{Im} \alpha$ and $\operatorname{Im}\left(\gamma^{-1} \beta\right)=\operatorname{Im} \beta$. Hence, $\gamma^{-1} \beta \in \operatorname{PAut}\left(P_{n}\right)$ maps each maximal interval $J$ of $\operatorname{Im} \alpha$ into a maximal interval $J \gamma^{-1} \beta$ of $\operatorname{Im} \beta$, thus defining a bijection $\sigma\left(J \mapsto J \sigma=J \gamma^{-1} \beta\right)$ from the set of maximal intervals of $\operatorname{Im} \alpha$ into the set of maximal intervals of $\operatorname{Im} \beta$. Let $J$ be a maximal interval of $\operatorname{Im} \alpha$. Then $(J \sigma) \beta^{-1}=J \gamma^{-1} \beta \beta^{-1}=J \gamma^{-1}=J \alpha^{-1} \alpha \gamma^{-1}=\left(J \alpha^{-1}\right) \alpha \gamma^{-1}$. Since $\alpha \gamma^{-1} \in$ $\operatorname{PAut}\left(P_{n}\right)$, we may deduce that $\tau_{\alpha}(J) \in\left\{\tau_{\beta}(J \sigma), \tau_{\beta}(J \sigma)^{R}\right\}$. Therefore $\alpha$ and $\beta$ have similar type.

Conversely, let $\alpha, \beta \in \operatorname{IEnd}\left(P_{n}\right)$ be such that $\alpha$ and $\beta$ have similar type. Then, by Lemma 1 , we have directly $\alpha \mathscr{J} \beta$, as required.

We finish this section with the description of Green's relation $\mathscr{J}$ of PAut $\left(P_{n}\right)$, which follows immediately from Lemma 1 and Proposition 3.

Corollary 2 Let $\alpha, \beta \in \operatorname{PAut}\left(P_{n}\right)$. Then $\alpha \mathscr{J} \beta$ if and only if $\alpha$ and $\beta$ have similar type.

Observe that the type of a maximal interval of the image of an element of PAut $\left(P_{n}\right)$ is always a unitary sequence which we can identify with the size of the interval taken. Therefore, two elements $\alpha$ and $\beta$ of $\operatorname{PAut}\left(P_{n}\right)$ have similar type if and only if they have maximal intervals of their images with the same size and with the same number of occurrences.

\section{Cardinality}

Let $n \in \mathbb{N}$ and $\bar{n}=\{1, \ldots, n\}$. We will determine the cardinality of $\operatorname{PAut}\left(P_{n}\right)$ as well as of IEnd $\left(P_{n}\right)$. For this, we need some technical notations.

Let $A \in\{0,1\}^{n}$ and let $A(p)$ denotes the element on the position $p$ in $A$. Further, let $A(0)=A(n+1)=0$.

Let $R_{A}=\{p \in \bar{n} \mid A(p-1)=0$ and $A(p)=1\}$ and $r_{A}=\left|R_{A}\right|$.

Let $s_{A}=\sum_{p=1}^{n} A(p)$.

Let $z(1)=1, z(2)=r_{A}$ and

$$
q_{A, i}=\left\{\begin{array}{cl}
\left(\begin{array}{c}
n-s_{A}+z(i) \\
r_{A}
\end{array}\right) & \text { if } A(p) \neq 0, \text { for some } p \in \bar{n} \\
1 & \text { otherwise },
\end{array}\right.
$$


for $i \in\{1,2\}$.

Let $t_{A, i}=\left(r_{A} !\right) q_{A, i}$, for $i \in\{1,2\}$ and let $T_{A}=\left|\left\{p \in R_{A} \mid A(p+1)=1\right\}\right|$.

Theorem 1 One has

$$
\mid \text { PAut }\left(P_{n}\right) \mid=\sum_{A \in\{0,1\}^{n}} 2^{T_{A}} t_{A, 1} \text { and } \mid \text { IEnd }\left(P_{n}\right) \mid=\sum_{A \in\{0,1\}^{n}} 2^{T_{A}} t_{A, 2} .
$$

Proof The domain of an injective endomorphism on $P_{n}$ is a subset of $\bar{n}$. For each $A \in\{0,1\}^{n}$, let $A^{*}$ be the subset of $\bar{n}$ with $x \in A^{*}$ if and only if $A(x)=1$. In particular, by $A \longmapsto A^{*}$, a bijection between $\{0,1\}^{n}$ and the powerset of $\bar{n}$, i.e. between $\{0,1\}^{n}$ and the possible domains of injective endomorphisms on $P_{n}$, is given. Let $A \in\{0,1\}^{n}$.

First, we suppose that $A \neq(0,0, \ldots, 0)$. Then $A^{*}$ consists of $r_{A}$ maximal intervals $A_{1}<A_{2}<\cdots<A_{r_{A}}$ of $A^{*}$. For $i \in\left\{1, \ldots, r_{A}\right\}$, let $p_{i}$ be the minimal element in the set $A_{i}$. So, we have $A\left(p_{i}-1\right)=0$ and $A\left(p_{i}\right)=1$, for $i \in\left\{1, \ldots, r_{A}\right\}$. This provides $R_{A}=\left\{p_{i} \mid i \in\left\{1, \ldots, r_{A}\right\}\right\}$. Moreover, we have $s_{A}=\left|A^{*}\right|$.

An injective endomorphism on $P_{n}$ with domain $A^{*}$ has the form

$$
\left(\begin{array}{llll}
A_{1} & A_{2} & \cdots & A_{r_{A}} \\
B_{1} & B_{2} & \cdots & B_{r_{A}}
\end{array}\right)
$$

where $B_{1}, \ldots, B_{r_{A}}$ are intervals. We observe that for each permutation $\sigma$ on $\left\{1, \ldots, r_{A}\right\}$, there is a possible image sequence $B_{1}, \ldots, B_{r_{A}}$ such that $B_{1 \sigma}<B_{2 \sigma}<$ $\cdots<B_{r_{A} \sigma}$, i.e. there are $r_{A}$ ! possibilities in which the intervals $B_{1}, \ldots, B_{r_{A}}$ are ordered. If the image sequence $B_{1}, \ldots, B_{r_{A}}$ is ordered by $B_{1 \sigma}<B_{2 \sigma}<\cdots<B_{r_{A} \sigma}$, for some permutation $\sigma$ on $\left\{1, \ldots, r_{A}\right\}$, then there are still $n-s_{A}$ elements being not in the image of an injective endomorphism. If we restricted us to partial automorphisms then there are $b_{1}, \ldots, b_{r_{A}-1} \in \bar{n}$ such that $B_{1 \sigma}<b_{1}<B_{2 \sigma}<b_{2}<\cdots<b_{r_{A}-1}<$ $B_{r_{A} \sigma}$ and so there are still $n-s_{A}-r_{A}+1$ elements being not in the image of a partial automorphism. These remaining elements can be distributed before or after the $B_{i}$ 's, i.e. at $r_{A}+1$ places. The number of all these possibilities is

$$
\left(\begin{array}{c}
\left(r_{A}+1\right)+\left(n-s_{A}\right)-1 \\
n-s_{A}
\end{array}\right)=\left(\begin{array}{c}
r_{A}+n-s_{A} \\
n-s_{A}
\end{array}\right)=\left(\begin{array}{c}
r_{A}+n-s_{A} \\
r_{A}
\end{array}\right)=q_{A, 2}
$$

for injective endomorphism and

$$
\left(\begin{array}{c}
\left(r_{A}+1\right)+\left(n-s_{A}-r_{A}+1\right)-1 \\
n-s_{A}-r_{A}+1
\end{array}\right)=\left(\begin{array}{c}
n-s_{A}+1 \\
n-s_{A}-r_{A}+1
\end{array}\right)=\left(\begin{array}{c}
n-s_{A}+1 \\
r_{A}
\end{array}\right)=q_{A, 1}
$$

if we only consider partial automorphisms. In other words, we have $q_{A, 2}\left(r_{A} !\right)=t_{A, 2}$ and $q_{A, 1}\left(r_{A} !\right)=t_{A, 1}$ possibilities for the intervals $B_{1}, \ldots, B_{r_{A}}$, whenever $A^{*}$ (with the partition $A_{1}<\cdots<A_{r_{A}}$ ) is the domain of an injective endomorphism and of a partial automorphism, respectively. For $i \in\left\{1, \ldots, r_{A}\right\}$, if $\left|A_{i}\right| \geq 2$ then we have to consider two cases, namely $\left(\begin{array}{c}A_{i} \\ B_{i}\end{array}\right)$ is order-preserving or order-reversing. In order to 
realize it, we consider the cardinality $T_{A}$ of the set $D_{A}=\left\{i \in\left\{1, \ldots, r_{A}\right\}|| A_{i} \mid \geq 2\right\}$, i.e. $T_{A}=\left|D_{A}\right|$. So, we have still to consider $2^{T_{A}}$ possibilities, whenever the intervals $B_{1}, \ldots, B_{r_{A}}$ are already fixed. Observe that $D_{A}=\left\{p \in R_{A} \mid A(p+1)=1\right\}$.

Thus, there are $2^{T_{A}} t_{A, 2}$ injective endomorphisms and $2^{T_{A}} t_{A, 1}$ partial automorphisms on $P_{n}$ with domain $A^{*}$.

Next, suppose that $A=(0,0, \ldots, 0)$. Then, there exists exactly one injective endomorphism on $P_{n}$ with the domain $A^{*}=\emptyset$, namely the empty transformation. In this case, we have $q_{A, 1}=q_{A, 2}=1$ and $r_{A}=T_{A}=0$. Hence, $t_{A, 1}=t_{A, 2}=$ $q_{A, 1}\left(r_{A} !\right)=1(0 !)=1,2^{T_{A}}=2^{0}=1$ and $2^{T_{A}} t_{A, 1}=2^{T_{A}} t_{A, 2}=1$.

We conclude that $\left|\operatorname{PAut}\left(P_{n}\right)\right|=\sum_{A \in\{0,1\}^{n}} 2^{T_{A}} t_{A, 1}$ and $\left|\operatorname{IEnd}\left(P_{n}\right)\right|=\sum_{A \in\{0,1\}^{n}} 2^{T_{A}} t_{A, 2}$, as required.

\section{Generators and rank}

In this section we present the main results of this paper. We are referring to the calculation of the ranks of $\operatorname{PAut}\left(P_{n}\right)$ and $\operatorname{IEnd}\left(P_{n}\right)$. In both cases, we proceed by determining a generating set of minimal size.

It is clear that PAut $\left(P_{1}\right)=\{\mathrm{id}, \emptyset\}$ is a generating set of minimal size of $\operatorname{PAut}\left(P_{1}\right)=$ $\operatorname{IEnd}\left(P_{1}\right)$, where $\varnothing$ is the empty transformation. Moreover, it is easy to verify that

$$
\mathscr{G}=\left\{\left(\begin{array}{ll}
1 & 2 \\
2 & 1
\end{array}\right),\left(\begin{array}{l}
1 \\
1
\end{array}\right)\right\}
$$

is a generating set of minimal size of

$$
\operatorname{PAut}\left(P_{2}\right)=\operatorname{IEnd}\left(P_{2}\right)=\left\{\mathrm{id},\left(\begin{array}{ll}
1 & 2 \\
2 & 1
\end{array}\right),\left(\begin{array}{l}
1 \\
1
\end{array}\right),\left(\begin{array}{l}
1 \\
2
\end{array}\right),\left(\begin{array}{l}
2 \\
1
\end{array}\right),\left(\begin{array}{l}
2 \\
2
\end{array}\right), \emptyset\right\} .
$$

This shows that

$$
\operatorname{rank} \operatorname{PAut}\left(P_{1}\right)=\operatorname{rank} \operatorname{IEnd}\left(P_{1}\right)=\operatorname{rank} \operatorname{PAut}\left(P_{2}\right)=\operatorname{rank} \operatorname{IEnd}\left(P_{2}\right)=2
$$

Next, let $n \geq 3$ and define

$$
\tau=\left(\begin{array}{lrrrr}
1 & 2 & \cdots & n-1 & n \\
n & n-1 & \cdots & 2 & 1
\end{array}\right)
$$

and

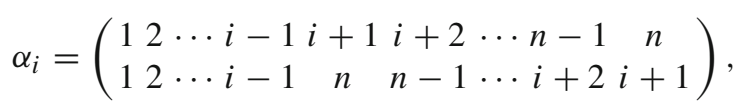

for $i=1,2, \ldots, n$. 
Let

$$
\mathscr{A}= \begin{cases}\left\{\tau, \alpha_{1}, \alpha_{2}\right\} & \text { if } n=3 \\ \{\tau\} \cup\left\{\alpha_{i} \mid i=1,2, \ldots, n-2\right\} & \text { if } n \geq 4\end{cases}
$$

First, we will show that $\mathscr{A}$ is a generating set of $\operatorname{PAut}\left(P_{n}\right)$. To accomplish this aim we start by proving a series of lemmas.

Lemma 2 One has $\left\{\alpha_{i} \mid i=n-1, n\right\} \subseteq\langle\mathscr{A}\rangle$.

Proof The proof follows immediately from the relations $\alpha_{i}=\tau \alpha_{n-i+1}^{2} \tau$, for $i=$ $n-1, n$.

Let

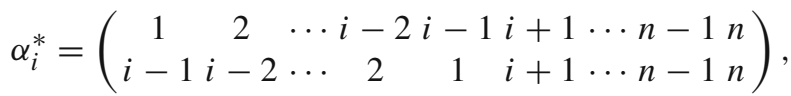

for $i=1,2, \ldots, n$.

Lemma 3 One has $\alpha_{i}^{*} \in\langle\mathscr{A}\rangle$, for $i=1,2, \ldots, n$.

Proof We have $\alpha_{i}^{*}=\alpha_{i} \tau \alpha_{n-i+1} \tau \alpha_{i}$, whence $\alpha_{i}^{*} \in\langle\mathscr{A}\rangle$, for $i=1,2, \ldots, n$.

Let

$$
\varepsilon_{i, j}=\left(\begin{array}{ll}
1 & 2 \cdots i-1 i+1 \cdots j-1 j+1 \cdots n \\
1 & 2 \cdots i-1 i+1 \cdots j-1 j+1 \cdots n
\end{array}\right),
$$

for $1 \leq i<i+1<j \leq n$.

Lemma 4 One has $\varepsilon_{i, j} \in\langle\mathscr{A}\rangle$, for $1 \leq i<i+1<j \leq n$.

Proof We have $\varepsilon_{i, j}=\alpha_{i}^{2} \alpha_{j}^{2}$, whence $\varepsilon_{i, j} \in\langle\mathscr{A}\rangle$, for $1 \leq i<i+1<j \leq n$.

Let

$$
\varepsilon_{i, j}^{*}=\left(\begin{array}{cccc}
1 & 2 \cdots i-1 i+1 i+2 \cdots j-2 j-1 j+1 \cdots n \\
1 & 2 \cdots i-1 j-1 j-2 \cdots i+2 i+1 j+1 \cdots n
\end{array}\right),
$$

for $1 \leq i<i+1<j \leq n$.

Lemma 5 One has $\varepsilon_{i, j}^{*} \in\langle\mathscr{A}\rangle$, for $1 \leq i<i+1<j \leq n$.

Proof We have $\varepsilon_{i, j}^{*}=\varepsilon_{i, j} \alpha_{j}^{*} \alpha_{j-i}^{*} \alpha_{j}^{*}$, which implies $\varepsilon_{i, j}^{*} \in\langle\mathscr{A}\rangle$, for $1 \leq i<i+1<$ $j \leq n$. 
Define $\alpha_{0}=\tau, \alpha_{n+1}=\mathrm{id}, \varepsilon_{0, n+1}^{*}=\tau, \varepsilon_{0, j}^{*}=\alpha_{j}^{*}$, for $j=2, \ldots, n$, and $\varepsilon_{i, n+1}^{*}=\alpha_{i}$, for $i=1, \ldots, n-1$.

Let

$$
\rho_{i, j}^{+}=\left(\begin{array}{lll}
1 & 2 \cdots i-1 i+2 \cdots j & j+2 \cdots n \\
1 & 2 \cdots i-1 i+1 \cdots j-1 j+2 \cdots n
\end{array}\right)
$$

for $0 \leq i<i+2<j \leq n$.

Lemma 6 One has $\rho_{i, j}^{+} \in\langle\mathscr{A}\rangle$, for $0 \leq i<i+2<j \leq n$.

Proof We have $\rho_{i, j}^{+}=\alpha_{i}^{2} \alpha_{i+1}^{2} \alpha_{j+1}^{2} \varepsilon_{i, j+1}^{*} \varepsilon_{i, j}^{*}$, whence $\rho_{i, j}^{+} \in\langle\mathscr{A}\rangle$, for $0 \leq i<i+2<$ $j \leq n$.

Let

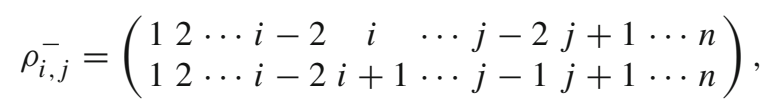

for $1 \leq i<i+2<j \leq n+1$.

Lemma 7 One has $\rho_{i, j}^{-} \in\langle\mathscr{A}\rangle$, for $1 \leq i<i+2<j \leq n+1$.

Proof We have $\rho_{i, j}^{-}=\alpha_{i-1}^{2} \alpha_{j-1}^{2} \alpha_{j}^{2} \varepsilon_{i-1, j}^{*} \varepsilon_{i, j}^{*}$, which implies $\rho_{i, j}^{-} \in\langle\mathscr{A}\rangle$, for $1 \leq i<$ $i+2<j \leq n+1$.

Now, we are prepared to prove that $\mathscr{A}$ is a generating set of the monoid $\operatorname{PAut}\left(P_{n}\right)$.

Proposition 4 One has $\operatorname{PAut}\left(P_{n}\right)=\langle\mathscr{A}\rangle$.

Proof We will perform this proof by using a recurring construction. First, for an arbitrary element $\alpha$ of PAut $\left(P_{n}\right)$, we set some notations. Denote by $I_{1}^{\alpha}, I_{2}^{\alpha}, \ldots, I_{k}^{\alpha}$ the maximal intervals of Dom $\alpha$ such that

$$
I_{1}^{\alpha}<I_{2}^{\alpha}<\cdots<I_{k}^{\alpha} .
$$

Let $J_{r}^{\alpha}=I_{r}^{\alpha} \alpha$, for $r=1, \ldots, k$. Then $J_{1}^{\alpha}, J_{2}^{\alpha}, \ldots, J_{k}^{\alpha}$ are the maximal intervals of $\operatorname{Im} \alpha$. Denote by $\sigma_{\alpha}$ the permutation of $\{1,2, \ldots, k\}$ such that

$$
J_{1 \sigma_{\alpha}}^{\alpha}<J_{2 \sigma_{\alpha}}^{\alpha}<\cdots<J_{k \sigma_{\alpha}}^{\alpha}
$$

Now, fix $\alpha \in \operatorname{PAut}\left(P_{n}\right)$. Let $I=\bar{n} \backslash$ Dom $\alpha$ and define $\beta=\prod_{i \in I} \alpha_{i}^{2}$ (observe that $\alpha_{i}^{2}, i \in \bar{n}$, is an idempotent and idempotents commute). Clearly, Dom $\beta=\operatorname{Dom} \alpha$.

Let $s$ be the least number $r \in\{1, \ldots, k\}$ such that $r \sigma_{\alpha} \neq r \sigma_{\beta}$. Let $t$ be the minimal element in the set $J_{s \sigma_{\beta}}^{\beta}$ and $q$ be the maximal element of $J_{s \sigma_{\alpha} \sigma_{\beta}}^{\beta}$. Then, we put

$$
\beta=\beta \varepsilon_{t-1, q+1}^{*}
$$


(i.e. we define a new $\beta$ as being $\beta \varepsilon_{t-1, q+1}^{*}$; below we will made similar variables's redefinitions). Then either $r \sigma_{\alpha}=r \sigma_{\beta}$, for all $r \in\{1, \ldots, k\}$, or the least number $r \in\{1, \ldots, k\}$ such that $r \sigma_{\alpha} \neq r \sigma_{\beta}$ is greater than $s$.

We repeat the procedure until $r \sigma_{\alpha}=r \sigma_{\beta}$ for all $r \in\{1, \ldots, k\}$.

Further, we put $\gamma=\beta$ and let $u$ be the least number $p \in\{1, \ldots, k\}$ such that $\left.\gamma\right|_{I_{p \sigma \gamma}^{\alpha}} ^{\alpha} \neq\left.\alpha\right|_{I_{p \sigma \gamma}^{\alpha}} ^{\alpha}$.

If $\left.\operatorname{Im} \gamma\right|_{I_{u \sigma \gamma}^{\alpha}} ^{\alpha}=\left.\operatorname{Im} \alpha\right|_{I_{u \sigma \gamma}^{\alpha}}$ then we put

$$
\gamma=\gamma \varepsilon_{a, b}^{*},
$$

where $a$ and $b$ are the greatest and respectively the least number with $a<J_{u \sigma_{\gamma}}^{\gamma}<b$.

If $\left.\operatorname{Im} \gamma\right|_{I_{u \sigma \gamma}^{\alpha}} ^{\alpha} \neq\left.\operatorname{Im} \alpha\right|_{I_{u \sigma_{\gamma}}^{\alpha}}$ then there exist $x, y \in \bar{n}$ such that $\left.\operatorname{Im} \alpha\right|_{I_{u \sigma \gamma}^{\alpha}}=\{x, \ldots, z\}$ and either $\left.\operatorname{Im} \gamma\right|_{I_{u \sigma \gamma}^{\alpha}} ^{\alpha}=\{x-y, \ldots, z-y\}$ or $\left.\operatorname{Im} \gamma\right|_{I_{u \sigma_{\gamma}}^{\alpha}}=\{x+y, \ldots, z+y\}$, where $z=x+\left|I_{u \sigma_{\gamma}}^{\alpha}\right|-1$.

First, suppose that $\left.\operatorname{Im} \gamma\right|_{I_{u \sigma_{\gamma}}^{\alpha}}=\{x-y, \ldots, z-y\}$. Then, there exists $j \in \bar{n}$ with $j>J_{u \sigma_{\gamma}}^{\gamma}$ such that $j, j+1 \notin \operatorname{Im} \gamma$. In this case, we put

$$
\gamma=\gamma \rho_{x-y, j+1}^{-}
$$

On the other hand, admit that $\left.\operatorname{Im} \gamma\right|_{I_{u \sigma_{\gamma}}^{\alpha}}=\{x+y, \ldots, z+y\}$. Then, there exists $j<J_{u \sigma_{\gamma}}^{\gamma}$, with $j>J_{p \sigma_{\gamma}}^{\gamma}$, for all $p<u$ such that $j-1, j \notin \operatorname{Im} \gamma$. In this case, we put

$$
\gamma=\gamma \rho_{j-1, z+y}^{+}
$$

After $y$ such steps, we obtain a transformation $\gamma$ such that $\left.\operatorname{Im} \gamma\right|_{I_{u \sigma_{\gamma}}^{\alpha}}=\left.\operatorname{Im} \alpha\right|_{I_{u \sigma_{\gamma}}^{\alpha}}$. If $\left.\gamma\right|_{I_{u \sigma_{\gamma}}^{\alpha}} \neq\left.\alpha\right|_{I_{u \sigma_{\gamma}}^{\alpha}}$ then we put

$$
\gamma=\gamma \varepsilon_{a, b}^{*},
$$

where $a$ and $b$ are the greatest and the least number, respectively, such that $a<J_{u \sigma_{\gamma}}^{\gamma}<$ b.

We repeat the procedure until $\gamma=\alpha$. Therefore, by Lemmas 2-7, we may deduce that $\alpha \in\langle\mathscr{A}\rangle$ and so $\mathscr{A}$ is a generating set of $\operatorname{PAut}\left(P_{n}\right)$, as required.

Next, we will show that $\mathscr{A}$ is a generating set of $\operatorname{PAut}\left(P_{n}\right)$ of minimal size.

Let $G$ be a generating set of $\operatorname{PAut}\left(P_{n}\right)$.

First, notice that Dom $\tau=\bar{n}$. Moreover, for $\alpha \in \operatorname{PAut}\left(P_{n}\right)$, clearly, we have Dom $\alpha=\bar{n}$ if and only if $\alpha=\tau$ or $\alpha=$ id $=\tau^{2}$. Thus, it follows immediately that:

Lemma 8 One has $\tau \in G$.

Let

$$
A_{i}=\left\{\alpha \in \operatorname{PAut}\left(P_{n}\right) \mid \operatorname{Dom} \alpha=\bar{n} \backslash\{i\} \text { or } \operatorname{Dom} \alpha=\bar{n} \backslash\{n-i+1\}\right\},
$$


for $i=1, \ldots,\left\lceil\frac{n}{2}\right\rceil$.

Lemma 9 One has $\left|G \cap A_{i}\right| \geq 1$, for all $i \in\left\{1, \ldots,\left\lceil\frac{n}{2}\right\rceil\right\}$.

Proof Let $i \in\left\{1, \ldots,\left\lceil\frac{n}{2}\right\rceil\right\}$ and consider the transformation $\alpha_{i}$ defined previously. Notice that Dom $\alpha_{i}=\bar{n} \backslash\{i\}$ and so $\alpha_{i} \in A_{i}$. Let $\beta_{1}, \ldots, \beta_{k} \in G \backslash\{$ id $\}$ be such that $\alpha_{i}=\beta_{1} \cdots \beta_{k}$ and $\left\{\beta_{j}, \beta_{j+1}\right\} \neq\{\tau\}$, for $j=1, \ldots, k-1$. Since Dom $\alpha_{i}=$ $\operatorname{Dom}\left(\beta_{1} \cdots \beta_{k}\right) \subseteq \operatorname{Dom} \beta_{1}$, rank $\alpha_{i}=n-1$ and $\beta_{1} \neq \mathrm{id}$, we have $\operatorname{Dom} \beta_{1}=\operatorname{Dom} \alpha_{i}$ or $\beta_{1}=\tau$.

If Dom $\beta_{1}=\operatorname{Dom} \alpha_{i}$ then $\beta_{1} \in A_{i}$ and so $\beta_{1} \in G \cap A_{i}$.

On the other hand, suppose that $\beta_{1}=\tau$. In that case, since Dom $\alpha_{i}=$ $\operatorname{Dom}\left(\beta_{1} \cdots \beta_{k}\right) \subseteq \operatorname{Dom}\left(\tau \beta_{2}\right)$, rank $\alpha_{i}=n-1$ and $\beta_{2} \in G \backslash\{$ id, $\tau\}$, we have $\operatorname{Dom} \alpha_{i}=\operatorname{Dom}\left(\tau \beta_{2}\right)$, whence $\operatorname{Dom} \beta_{2}=\bar{n} \backslash\{n-i+1\}$ and so $\beta_{2} \in G \cap A_{i}$.

Thus, in both cases, we have shown that $\left|G \cap A_{i}\right| \neq \emptyset$, as required.

Lemma 10 Let $n \geq 6$. Then $\left|G \cap A_{i}\right| \geq 2$, for all $i \in\left\{3, \ldots,\left\lfloor\frac{n}{2}\right\rfloor\right\}$.

Proof First, observe that it is a routine matter to check that $\left|A_{i}\right|=16$, for all $i \in$ $\left\{3, \ldots,\left\lfloor\frac{n}{2}\right\rfloor\right\}$. Recall also that $\tau \in G$, by Lemma 8 .

Now, assume by contradiction that $\left|G \cap A_{i}\right|<2$, for some $i \in\left\{3, \ldots,\left\lfloor\frac{n}{2}\right\rfloor\right\}$. Then, by Lemma 9, we have $G \cap A_{i}=\{\alpha\}$, for some $\alpha \in \operatorname{PAut}\left(P_{n}\right)$. Without loss of generality, we may suppose that $\operatorname{Dom} \alpha=\bar{n} \backslash\{i\}$. Hence, we have two cases:

Case $1 . \operatorname{Im} \alpha=\bar{n} \backslash\{i\}$. Then, as $\alpha^{3}=\alpha$ and rank $\alpha \tau \alpha=n-2$, we have

$$
\langle G\rangle \cap A_{i}=\left\{\alpha, \alpha^{2}, \alpha \tau, \tau \alpha, \alpha^{2} \tau, \tau \alpha^{2}, \tau \alpha \tau, \tau \alpha^{2} \tau\right\} \neq A_{i},
$$

which is a contradiction (since $G$ is a generating set of PAut $\left(P_{n}\right)$ ).

Case 2. $\operatorname{Im} \alpha=\bar{n} \backslash\{n-i+1\}$. In this case, as $(\alpha \tau)^{2}=\left.\mathrm{id}\right|_{\operatorname{Dom} \alpha},(\tau \alpha)^{2}=\left.\mathrm{id}\right|_{\operatorname{Im} \alpha}$ and rank $\alpha^{2}=n-2$, we obtain

$$
\langle G\rangle \cap A_{i}=\left\{\alpha, \alpha \tau, \tau \alpha, \alpha \tau \alpha, \tau \alpha \tau,(\alpha \tau)^{2},(\tau \alpha)^{2}, \tau(\alpha \tau)^{2}\right\} \neq A_{i},
$$

which again is a contradiction, as required.

Now, as a consequence of Proposition 4 and Lemmas 8-10, we may prove the first of our main results:

Theorem 2 The rank of PAut $\left(P_{3}\right)$ is equal to 3 and, for $n \geq 4$, the rank of $\operatorname{PAut}\left(P_{n}\right)$ is equal to $n-1$.

Proof By Proposition 4, the set $\mathscr{A}$ generates PAut $\left(P_{n}\right)$. Thus,

$$
\operatorname{rank} \operatorname{PAut}\left(P_{n}\right) \leq|\mathscr{A}|= \begin{cases}3 & \text { if } n=3 \\ n-1 & \text { if } n \geq 4\end{cases}
$$

Let $G$ be any generating set of PAut $\left(P_{n}\right)$. By Lemmas 8 and 9 , the transformation $\tau$ and $\left\lceil\frac{n}{2}\right\rceil$ pairwise different transformations of rank $n-1$ are in $G$. Thus, $|G| \geq 1+\left\lceil\frac{n}{2}\right\rceil$. In particular, we have $|G| \geq 3$, if $n=3,4$, and $|G| \geq 4$, if $n=5$. If $n \geq 6$ then, 
by Lemma 10, there exist $\left\lfloor\frac{n}{2}\right\rfloor-2$ additional pairwise different transformations with rank $n-1$ in $G$. This shows that

$$
\operatorname{rank} \operatorname{PAut}\left(P_{n}\right) \geq \begin{cases}3 & \text { if } n=3 \\ n-1 & \text { if } n \geq 4\end{cases}
$$

as required.

Next, we calculate the rank of the monoid $\operatorname{IEnd}\left(P_{n}\right)$.

Define

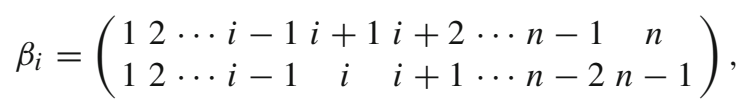

for $i=2, \ldots, n-1$, and let $\mathscr{B}=\mathscr{A} \cup\left\{\beta_{i} \mid i=2, \ldots,\left\lceil\frac{n}{2}\right\rceil\right\}$.

Lemma 11 One has $\left\{\beta_{i} \mid i=2, \ldots, n-1\right\} \subseteq\langle\mathscr{B}\rangle$.

Proof For $i=2, \ldots,\left\lceil\frac{n}{2}\right\rceil$, we have $\beta_{i} \in \mathscr{B}$. Let $i=\left\lceil\frac{n}{2}\right\rceil+1, \ldots, n-1$ then $\beta_{i}=\tau \beta_{n-i+1} \alpha_{n}^{*}$.

Proposition 5 Let $\beta \in \operatorname{IEnd}\left(P_{n}\right) \backslash \operatorname{PAut}\left(P_{n}\right)$. Then

$$
\beta \in\left\langle\operatorname{PAut}\left(P_{n}\right) \cup\left\{\beta_{i} \mid i=2, \ldots, n-1\right\}\right\rangle .
$$

Proof Let $\beta \in \operatorname{IEnd}\left(P_{n}\right) \backslash \operatorname{PAut}\left(P_{n}\right)$. Then, it is easy to show that there exists a transformation $\delta \in \operatorname{PAut}\left(P_{n}\right)$ with $\operatorname{Dom} \delta=\operatorname{Im} \beta$ and $\operatorname{Im} \delta \subseteq\left\{1,2, \ldots,|\operatorname{Im} \beta|+\mathrm{m}_{\beta}-1\right\}$, where $\mathrm{m}_{\beta}$ is the number of the maximal intervals of $\operatorname{Im} \beta$.

Define $\bar{\beta}=\beta \delta$. It is clear that $\operatorname{Dom} \bar{\beta}=\operatorname{Dom} \beta$.

Further, let $I$ be the set of all $x \in \operatorname{Dom} \bar{\beta}$ such that $x \bar{\beta}+1 \in \operatorname{Im} \bar{\beta}$ and $x \bar{\beta}+1 \notin$ $\{(x-1) \bar{\beta},(x+1) \bar{\beta}\}$. Clearly, $I \neq \emptyset$ since $\beta \notin \operatorname{PAut}\left(P_{n}\right)$. We let $I=\left\{i_{1}, \ldots, i_{k}\right\}$ be such that $i_{1} \bar{\beta}<i_{2} \bar{\beta}<\cdots<i_{k} \bar{\beta}$.

Let $X_{1}, X_{2}, \ldots, X_{k+1}$ be the partition of Dom $\bar{\beta}$ such that

$$
X_{r}=\left\{x \in \operatorname{Dom} \bar{\beta} \mid i_{r-1} \bar{\beta}<x \bar{\beta} \leq i_{r} \bar{\beta}\right\}
$$

for $r \in\{1,2, \ldots, k+1\}$, where $i_{0} \bar{\beta}=0$ and $i_{k+1} \bar{\beta}=n$.

Let $\beta^{*}$ be the transformation defined by $x \beta^{*}=x \bar{\beta}+r-1$, for all $x \in X_{r}$ and $r=1,2, \ldots, k+1$. It is clear that $\operatorname{Dom} \beta^{*}=\operatorname{Dom} \bar{\beta}=\operatorname{Dom} \beta$ and $\beta^{*} \in \operatorname{IEnd}\left(P_{n}\right)$.

First, we show $\beta^{*} \in \operatorname{PAut}\left(P_{n}\right)$. Let $u \in \bar{n}$ be such that $u, u+1 \in \operatorname{Im} \beta^{*}$. Then there exist $a \in X_{r_{1}}$ and $b \in X_{r_{2}}$ such that $a \bar{\beta}+r_{1}-1=u$ and $b \bar{\beta}+r_{2}-1=u+1$, for some $r_{1}, r_{2} \in\{1, \ldots, k+1\}$. In order to obtain a contradiction, assume that $r_{1} \neq r_{2}$.

Suppose that $r_{1}<r_{2}$. Then $X_{r_{1}} \bar{\beta}<X_{r_{2}} \bar{\beta}$ and so $a \bar{\beta}<b \bar{\beta}$. This implies $r_{1}+1 \leq r_{2}$ and $a \bar{\beta}+1 \leq b \bar{\beta}$, whence $b \bar{\beta}+r_{2} \geq a \bar{\beta}+1+r_{1}+1=a \bar{\beta}+r_{1}-1+3=u+3=$ $b \bar{\beta}+r_{2}-1+2=b \bar{\beta}+r_{2}+1$. Thus $b \bar{\beta} \geq b \bar{\beta}+1$, which is a contradiction.

On the other hand, suppose that $r_{1}>r_{2}$. Then $X_{r_{1}} \bar{\beta}>X_{r_{2}} \bar{\beta}$ and so $a \bar{\beta}>b \bar{\beta}$. This implies $r_{1} \geq r_{2}+1$ and $a \bar{\beta} \geq b \bar{\beta}+1$. Thus, we have $a \bar{\beta}+r_{1} \geq b \bar{\beta}+1+r_{2}+1=$ 
$b \bar{\beta}+r_{2}-1+3=u+1+3=a \bar{\beta}+r_{1}+3$, whence $a \bar{\beta} \geq a \bar{\beta}+3$, which is a contradiction.

Therefore, we have $r_{1}=r_{2}$. Then $u=a \bar{\beta}+r_{1}-1, u+1=b \bar{\beta}+r_{1}-1$ and so $a, b \in X_{r_{1}}$. This implies $b \bar{\beta}+r_{1}=b \bar{\beta}+r_{1}-1+1=u+1+1=$ $a \bar{\beta}+r_{1}-1+2=a \bar{\beta}+r_{1}+1$, i.e. $b \bar{\beta}=a \bar{\beta}+1$. Thus $a \bar{\beta}+1 \in \operatorname{Im} \bar{\beta}$, since $b \in \operatorname{Dom} \bar{\beta}$. Assume $a \bar{\beta}+1 \notin\{(a-1) \bar{\beta},(a+1) \bar{\beta}\}$. Then $a \in I$ and so $a=i_{r_{1}}$ and $b \bar{\beta} \leq a \bar{\beta}$, since $a, b \in X_{r_{1}}$. Hence, $a \bar{\beta}+1=b \bar{\beta} \leq a \bar{\beta}$, which is a contradiction. Thus, $b \bar{\beta}=a \bar{\beta}+1 \in\{(a-1) \bar{\beta},(a+1) \bar{\beta}\}$ and so we obtain $b \in\{a-1, a+1\}$.

This shows that $\beta^{*} \in \operatorname{PAut}\left(P_{n}\right)$.

Finally, we show that $\beta=\beta^{*} \beta_{i_{1} \bar{\beta}+1} \beta_{i_{2} \bar{\beta}+1} \cdots \beta_{i_{k} \bar{\beta}+1} \delta^{-1}$, from which follows that

$$
\beta \in\left\langle\operatorname{PAut}\left(P_{n}\right) \cup\left\{\beta_{i} \mid i=2, \ldots, n-1\right\}\right\rangle .
$$

Since $\bar{\beta} \delta^{-1}=\beta \delta \delta^{-1}=\beta$ id $\left.\right|_{\operatorname{Dom} \delta}=\beta$ id $\left.\right|_{\operatorname{Im} \beta}=\beta$, it suffices to show that $\bar{\beta}=$ $\beta^{*} \beta_{i_{1} \bar{\beta}+1} \beta_{i_{2} \bar{\beta}+1} \cdots \beta_{i_{k} \bar{\beta}+1}$.

We proceed by showing that

$$
\begin{aligned}
x & \beta^{*} \beta_{i_{1} \bar{\beta}+1} \beta_{i_{2} \bar{\beta}+1} \cdots \beta_{i_{s} \bar{\beta}+1} \\
& =\left\{\begin{array}{l}
x \bar{\beta}, \quad \text { if } x \in X_{1} \cup \cdots \cup X_{s} \\
x \bar{\beta}+r-1-s,
\end{array}\right.
\end{aligned}
$$

by induction on $1 \leq s \leq k$.

Let $s=1$. Then

$$
\begin{aligned}
& x \beta^{*} \beta_{i_{1} \bar{\beta}+1}=(x \bar{\beta}+r-1) \beta_{i_{1} \bar{\beta}+1} \\
& \quad=\left\{\begin{array}{lr}
(x \bar{\beta}) \beta_{i_{1} \bar{\beta}+1}=x \bar{\beta} & \text { if } x \in X_{1}, \\
x \bar{\beta}+r-1-1=x \bar{\beta}+r-1-s & \text { if } x \in X_{r} \text { for some } r>1, \\
& \text { since } x \bar{\beta} \geq i_{1} \bar{\beta}+1 .
\end{array}\right.
\end{aligned}
$$

Assume that the above expression is true for some $s<k$. We will prove it for $s+1$. Let $x \in X_{1} \cup \cdots \cup X_{s+1}$. If $x \notin X_{s+1}$ then $x \beta^{*} \beta_{i_{1} \bar{\beta}+1} \cdots \beta_{i_{s} \bar{\beta}+1}=x \bar{\beta}$, by the induction hypothesis and $(x \bar{\beta}) \beta_{i_{s+1} \bar{\beta}+1}=x \bar{\beta}$, since $x \bar{\beta}<i_{s+1} \bar{\beta}+1$.

If $x \in X_{s+1}$ then $x \beta^{*} \beta_{i_{1} \bar{\beta}+1} \cdots \beta_{i_{s} \bar{\beta}+1}=x \bar{\beta}+s+1-1-s=x \bar{\beta}$, by the induction hypothesis and $(x \bar{\beta}) \beta_{i_{s+1} \bar{\beta}+1}=x \bar{\beta}$, since $x \bar{\beta}<i_{s+1} \bar{\beta}+1$.

Now, let $x \in X_{r}$ for some $r \in\{s+2, \ldots, k+1\}$. Then

$$
\begin{aligned}
\left(x \beta^{*} \beta_{i_{1} \bar{\beta}+1} \cdots \beta_{i_{s} \bar{\beta}+1}\right) \beta_{i_{s+1} \bar{\beta}+1} & =(x \bar{\beta}+r-1-s) \beta_{i_{s+1} \bar{\beta}+1}=(x \bar{\beta}+r-1-s)-1 \\
& =x \bar{\beta}+r-1-(s+1),
\end{aligned}
$$

since $x \bar{\beta}>i_{s+1} \bar{\beta}+1$, which completes the proof.

From Proposition 4, Lemma 11 and Proposition 5, we obtain immediately:

Corollary 3 One has $\operatorname{IEnd}\left(P_{n}\right)=\langle\mathscr{B}\rangle$. 
Now, we will prove that $\mathscr{B}$ is a generating set of $\operatorname{IEnd}\left(P_{n}\right)$ of minimal size. We start by presenting a series of five lemmas.

Let $G^{\prime}$ be a generating set of $\operatorname{IEnd}\left(P_{n}\right)$.

Lemma 12 One has $\left|G^{\prime} \cap\left(\operatorname{IEnd}\left(P_{n}\right) \backslash \operatorname{PAut}\left(P_{n}\right)\right)\right| \geq\left\lceil\frac{n}{2}\right\rceil-1$.

Proof Let $2 \leq j \leq n-1$. Then $\beta_{j}=\gamma_{1} \cdots \gamma_{k}$, for some $k \geq 1$ and $\gamma_{1}, \ldots, \gamma_{k} \in G^{\prime}$. As rank $\beta_{j}=n-1$ then rank $\gamma_{i} \geq n-1$, for all $i=1, \ldots, k$, and there exists $i \in\{1, \ldots, k\}$ such that $\gamma_{i} \notin \operatorname{PAut}\left(P_{n}\right)$. Let $i$ be the least $r \in\{1, \ldots, k\}$ such as $\gamma_{r} \notin \operatorname{PAut}\left(P_{n}\right)$. Let $\gamma=\gamma_{1} \cdots \gamma_{i-1} \in \operatorname{PAut}\left(P_{n}\right)$ (with $\gamma=\mathrm{id}$ if $\left.i=1\right)$. Thus, $\beta_{j}=$ $\gamma \gamma_{i} \cdots \gamma_{k}$ implies $\gamma^{-1} \beta_{j}=\gamma^{-1} \gamma \gamma_{i} \cdots \gamma_{k}=\gamma_{i} \cdots \gamma_{k}\left(\right.$ since $\left.\gamma^{-1} \gamma=\left.\mathrm{id}\right|_{\operatorname{Dom} \gamma_{i}}\right)$.

We have rank $\gamma=n-1$ or rank $\gamma=n$. If $\operatorname{rank} \gamma=n-1$ then $\operatorname{Im} \gamma^{-1}=$ $\operatorname{Dom} \beta_{j}=\{1, \ldots, n\} \backslash\{j\}$. Thus Dom $\gamma=\{1, \ldots, n\} \backslash\{j\}$, whence $\operatorname{Im} \gamma=\operatorname{Dom} \beta_{j}$ or $\operatorname{Im} \gamma=\operatorname{Dom} \beta_{n-j+1}$, and so $\operatorname{Dom} \gamma_{i}=\operatorname{Im} \gamma=\operatorname{Dom} \beta_{j}$ or Dom $\gamma_{i}=\operatorname{Im} \gamma=$ $\operatorname{Dom} \beta_{n-j+1}$ (since rank $\gamma_{i}=n-1$ ). If rank $\gamma=n$ then $\gamma=$ id or $\gamma=\tau$. If $\gamma=$ id then $\operatorname{Dom} \gamma_{i}=\operatorname{Dom} \beta_{j}$. If $\gamma=\tau$ then $\operatorname{Dom} \gamma_{i}=\operatorname{Dom} \beta_{n-j+1}$. Note that $n-j+1 \geq\left\lceil\frac{n}{2}\right\rceil$.

Therefore, we must have in $G^{\prime}$ at least $\left\lceil\frac{n-2}{2}\right\rceil=\left\lceil\frac{n}{2}\right\rceil-1$ distinct elements of $\operatorname{IEnd}\left(P_{n}\right) \backslash \operatorname{PAut}\left(P_{n}\right)$.

Lemma 13 For $\alpha \in \operatorname{IEnd}\left(P_{n}\right)$ such that $\operatorname{Dom} \alpha \in\{\{1, \ldots, n-1\},\{2, \ldots, n\}\}$, we have $\alpha \in \operatorname{PAut}\left(P_{n}\right)$.

Proof It is a routine matter to verify that

$$
\alpha \in\left\{\alpha_{1}, \tau \alpha_{1}, \alpha_{1} \tau, \tau \alpha_{1} \tau, \alpha_{n}, \tau \alpha_{n}, \alpha_{n} \tau, \tau \alpha_{n} \tau\right\} \subseteq \operatorname{PAut}\left(P_{n}\right)
$$

as required.

Lemma 14 If $\alpha \in \operatorname{IEnd}\left(P_{n}\right) \backslash \operatorname{PAut}\left(P_{n}\right)$ has rank $n-1$, then $\operatorname{Im} \alpha \in\{\{1, \ldots, n-1\}$, $\{2, \ldots, n\}\}$.

Proof As rank $\alpha=n-1$, we conclude that $\alpha \in\left\{\beta_{i}, \tau \beta_{i}, \beta_{i} \tau, \tau \beta_{i} \tau \mid i=2, \ldots, n-1\right\}$. Let $i=2, \ldots, n-1$. Since $\operatorname{Im} \beta_{i}=\{1, \ldots, n-1\}$ and $\operatorname{Dom} \tau=\operatorname{Im} \tau=\bar{n}$, we obtain

$$
\operatorname{Im} \beta_{i}, \operatorname{Im}\left(\tau \beta_{i}\right), \operatorname{Im}\left(\beta_{i} \tau\right), \operatorname{Im}\left(\tau \beta_{i} \tau\right) \in\{\{1, \ldots, n-1\},\{2, \ldots, n\}\},
$$

whence $\operatorname{Im} \alpha \in\{\{1, \ldots, n-1\},\{2, \ldots, n\}\}$, as required.

Lemma 15 One has $\left\langle G^{\prime} \cap \operatorname{PAut}\left(P_{n}\right)\right\rangle=\operatorname{PAut}\left(P_{n}\right)$.

Proof First, notice that it is clear that $\tau \in G^{\prime}$.

On the other hand, let $\alpha$ be any transformation of PAut $\left(P_{n}\right)$ with $\operatorname{rank} \alpha=n-1$.

Then, there exist $\gamma_{1}, \ldots, \gamma_{k} \in G^{\prime}$ such that $\alpha=\gamma_{1} \cdots \gamma_{k}(k \geq 1)$. Assume that there exists $i \in\{1, \ldots, k\}$ such that $\gamma_{i} \notin \operatorname{PAut}\left(P_{n}\right)$. Let $i$ be the least index $r \in\{1, \ldots, k\}$ such that $\gamma_{r} \notin \operatorname{PAut}\left(P_{n}\right)$ and let $\gamma=\gamma_{1} \cdots \gamma_{i-1} \in \operatorname{PAut}\left(P_{n}\right)$ (with $\gamma=$ id if $i=1)$. Then $\alpha=\gamma \gamma_{i} \cdots \gamma_{k}$ implies $\gamma_{i} \cdots \gamma_{k}=\gamma^{-1} \gamma \gamma_{i} \cdots \gamma_{k}=\gamma^{-1} \alpha \in \operatorname{PAut}\left(P_{n}\right)$ 
(since $\gamma^{-1} \gamma=\left.\mathrm{id}\right|_{\operatorname{Dom} \gamma_{i}}$ ). Hence, we have $i<k$. We have $\gamma_{i+1} \cdots \gamma_{k} \notin\{\mathrm{id}, \tau\}$ (otherwise $\gamma_{i}=\gamma^{-1} \alpha\left(\gamma_{i+1} \cdots \gamma_{k}\right)^{-1} \in \operatorname{PAut}\left(P_{n}\right)$, which is a contradiction). Hence, $\operatorname{rank}\left(\gamma_{i+1} \cdots \gamma_{k}\right)=n-1$. Let $\lambda=\gamma_{i+1} \cdots \gamma_{k}$. Then Dom $\lambda=\operatorname{Im} \gamma_{i} \in\{\{1, \ldots, n-$ $1\},\{2, \ldots, n\}\}$, by Lemma 14. Therefore, we obtain $\lambda \in \operatorname{PAut}\left(P_{n}\right)$, by Lemma 13 . Thus, $\gamma^{-1} \alpha=\gamma_{i} \lambda$ implies that $\gamma_{i}=\gamma^{-1} \alpha \lambda^{-1} \in \operatorname{PAut}\left(P_{n}\right)$, which is a contradiction. Thus, $\gamma_{1}, \ldots, \gamma_{k} \in \operatorname{PAut}\left(P_{n}\right)$.

Therefore, in particular, we showed that $\mathscr{A} \subseteq\left\langle G^{\prime} \cap \operatorname{PAut}\left(P_{n}\right)\right\rangle$, and therefore, $\left\langle G^{\prime} \cap \operatorname{PAut}\left(P_{n}\right)\right\rangle=\operatorname{PAut}\left(P_{n}\right)$, by Proposition 4 .

Now, as an immediate consequence of Lemma 15 and Theorem 2, we have:

Lemma 16 One has $\left|G^{\prime} \cap \operatorname{PAut}\left(P_{n}\right)\right| \geq\left\{\begin{array}{lr}3 & \text { if } n=3 \\ n-1 & \text { if } n \geq 4\end{array}\right.$

Finally, we conclude with the presentation of our second main result.

Theorem 3 The rank of $\operatorname{IEnd}\left(P_{3}\right)$ is equal to 4 and, for $n \geq 4$, the rank of $\operatorname{IEnd}\left(P_{n}\right)$ is equal to $n+\left\lceil\frac{n}{2}\right\rceil-2$.

Proof By Corollary 3, we have

$$
\operatorname{rank} \operatorname{IEnd}\left(P_{n}\right) \leq|\mathscr{B}|=\left\{\begin{array}{lr}
3+1=4 & \text { if } n=3 \\
n-1+\left\lceil\frac{n}{2}\right\rceil-1=n+\left\lceil\frac{n}{2}\right\rceil-2 & \text { if } n \geq 4
\end{array}\right.
$$

On the other hand, by Lemmas 12 and 16 , we have

$$
\operatorname{rank} \operatorname{IEnd}\left(P_{n}\right) \geq\left\{\begin{array}{lr}
3+1=4 & \text { if } n=3 \\
n-1+\left\lceil\frac{n}{2}\right\rceil-1=n+\left\lceil\frac{n}{2}\right\rceil-2 & \text { if } n \geq 4,
\end{array}\right.
$$

as required.

Acknowledgements This work was produced, in part, during the visit of the first and third authors to CMA, FCT NOVA, Lisbon, in July 2019. The first author was supported by CMA through a visiting researcher fellowship.

\section{References}

1. Al-Kharousi, F., Kehinde, R., Umar, A.: On the semigroup of partial isometries of a finite chain. Commun. Algebra 44, 639-647 (2016)

2. Araújo, J., Bentz, W., Mitchell, J.D., Schneider, C.: The rank of the semigroup of transformations stabilising a partition of a finite set. Math. Proc. Camb. Philos. Soc. 159, 339-353 (2015)

3. Arworn, S.: An algorithm for the numbers of endomorphisms on paths. Discrete Math. 309, 94-103 (2009)

4. Arworn, S., Knauer, U., Leeratanavalee, S.: Locally strong endomorphisms of paths. Discrete Math. 308, 2525-2532 (2008)

5. Cicaló, S., Fernandes, V.H., Schneider, C.: Partial transformation monoids preserving a uniform partition. Semigroup Forum 90, 532-544 (2015)

6. Dimitrova, I., Fernandes, V.H., Koppitz, J., Quinteiro, T.M.: Ranks of monoids of endomorphisms of a finite undirected path. Bull. Malays. Math. Sci. Soc. 43, 1623-1645 (2015) 
7. Fan, S.: On end-regular bipartite graphs. In: Combinatorics and Graph Theory. Proceedings of the Spring School and International Conference on Combinatorics, pp. 117-130. World Scientific, Singapore (1993)

8. Fan, S.: The regularity of the endomorphism monoid of a split graph. Acta Math. Sin. 40, 419-422 (1997)

9. Fan, S.: Retractions of split graphs and End-orthodox split graphs. Discrete Math. 257, 161-164 (2002)

10. Fernandes, V.H.: Presentations for some monoids of partial transformations on a finite chain: a survey. In: Semigroups. Algorithms, Automata and Languages (Coimbra, 2001), pp. 363-378. World Scientific, River Edge, NJ (2002)

11. Fernandes, V.H., Honyam, P., Quinteiro, T.M., Singha, B.: On semigroups of endomorphisms of a chain with restricted range. Semigroup Forum 89, 77-104 (2014)

12. Fernandes, V.H., Honyam, P., Quinteiro, T.M., Singha, B.: On semigroups of orientation-preserving transformations with restricted range. Commun. Algebra 44, 253-264 (2016)

13. Fernandes, V.H., Koppitz, J., Musunthia, T.: The rank of the semigroup of all order-preserving transformations on a finite fence. Bull. Malays. Math. Sci. Soc. 42, 2191-2211 (2019)

14. Fernandes, V.H., Quinteiro, T.M.: On the ranks of certain monoids of transformations that preserve a uniform partition. Commun. Algebra 42, 615-636 (2014)

15. Fernandes, V.H., Sanwong, J.: On the rank of semigroups of transformations on a finite set with restricted range. Algebra Colloq. 21, 497-510 (2014)

16. Hou, H., Gu, R.: Split graphs whose completely regular endomorphisms form a monoid. Ars Comb. 127, 79-88 (2016)

17. Hou, H., Gu, R., Shang, Y.: The join of split graphs whose regular endomorphisms form a monoid. Commun. Algebra 42, 795-802 (2014)

18. Hou, H., Gu, R., Shang, Y.: The join of split graphs whose quasi-strong endomorphisms form a monoid. Bull. Austral. Math. Soc. 91, 1-10 (2015)

19. Hou, H., Luo, Y., Cheng, Z.: The endomorphism monoid of $\bar{P}_{n}$. Eur. J. Comb. 29, 1173-1185 (2008)

20. Hou, H., Luo, Y., Fan, S.: End-regular and end-orthodox joins of split graphs. Ars Comb. 105, 305-318 (2012)

21. Hou, H., Song, Y., Gu, R.: The join of split graphs whose completely regular endomorphisms form a monoid. De Gruyter Open Math. 15, 833-839 (2017)

22. Howie, J.M.: Fundamentals of Semigroup Theory. Clarendon Press, Oxford (1995)

23. Huisheng, P.: On the rank of the semigroup $T_{\rho}(X)$. Semigroup Forum 70, 107-117 (2005)

24. Knauer, U.: Algebraic Graph Theory: Morphisms, Monoids, and Matrices. De Gruyter, Berlin (2011)

25. Knauer, U., Wanichsombat, A.: Completely regular endomorphisms of split graphs. Ars Comb. 115, 357-366 (2014)

26. Li, W.: Split graphs with completely regular endomorphism monoids. J. Math. Res. Expos. 26, 253-263 (2006)

27. Li, W., Chen, J.: Endomorphism-regularity of split graphs. Eur. J. Comb. 22, 207-216 (2001)

28. Lu, D., Wu, T.: Endomorphism monoids of generalized split graphs. Ars Comb. 11, 357-373 (2013)

29. Marki, L.: Problems raised at the problem session of the colloquium on semigroups in Szeged, August 1987. Semigroup Forum 37, 367-373 (1988)

30. Michels, M.A., Knauer, U.: The congruence classes of paths and cycles. Discrete Math. 309, 5352-5359 (2009)

31. Pipattanajinda, N., Knauer, U., Gyurov, B., Panma, S.: The endomorphism monoids of ( $n-3)$-regular graphs of order $n$. Algebra Discrete Math. 22-2, 284-300 (2016)

32. Wilkeit, E.: Graphs with a regular endomorphism monoid. Arch. Math. 66, 344-352 (1996)

33. Zhao, P.: On the ranks of certain semigroups of orientation preserving transformations. Commun. Algebra 39, 4195-4205 (2011)

34. Zhao, P., Fernandes, V.H.: The ranks of ideals in various transformation monoids. Commun. Algebra 43, 674-692 (2015)

Publisher's Note Springer Nature remains neutral with regard to jurisdictional claims in published maps and institutional affiliations. 


\section{Authors and Affiliations}

\section{Dimitrova ${ }^{2}$ • V. H. Fernandes ${ }^{3}$. J. Koppitz ${ }^{1}$ - T. M. Quinteiro ${ }^{4,5}$}

$凶$ J. Koppitz

koppitz@math.bas.bg

I. Dimitrova

ilinka_dimitrova@swu.bg

V. H. Fernandes

vhf@fct.unl.pt

T. M. Quinteiro

teresa.melo@isel.pt

1 Institute of Mathematics and Informatics, Bulgarian Academy of Sciences, 1113 Sofia, Bulgaria

2 Department of Mathematics, Faculty of Mathematics and Natural Science, South-West University "Neofit Rilski”, 2700 Blagoevgrad, Bulgaria

3 CMA \& Departamento de Matemática, Nova School of Science and Technology, Universidade Nova de Lisboa, Monte da Caparica, 2829-516 Caparica, Portugal

4 Instituto Superior de Engenharia de Lisboa, 1950-062, Lisboa, Portugal

5 Present Address: CMA, Nova School of Science and Technology, Universidade Nova de Lisboa, Monte da Caparica, 2829-516 Caparica, Portugal 